\title{
Hip abductor neuromuscular capacity: A limiting factor in mediolateral balance control in older adults?
}

\author{
Mina Arvin ${ }^{a}$, Jaap H. van Dieën ${ }^{\text {a,* }}$, Gert S. Faber ${ }^{\text {a }}$, Mirjam Pijnappels ${ }^{\text {, }}$, \\ Marco J.M. Hoozemans ${ }^{\mathrm{a}, \mathrm{b}}$, Sabine M.P. Verschueren ${ }^{\mathrm{c}}$ \\ a MOVE Research Institute Amsterdam, Department of Human Movement Sciences, VU University Amsterdam, Amsterdam, The Netherlands \\ b CORAL - Centre for Orthopaedic Research Alkmaar, Orthopaedic Outpatient Department, Medical Centre Alkmaar, Alkmaar, The Netherlands \\ c Department of Rehabilitation Sciences, Faculty of Kinesiology and Rehabilitation Sciences, KU Leuven, Leuven, Belgium
}

\section{A R T I C L E I N F O}

\section{Article history:}

Received 12 November 2015

Received in revised form 4 April 2016

Accepted 31 May 2016

\section{Keywords:}

Elderly

Sensory input

Hip position sense

Hip abductor strength

Accidental falls

Postural balance

\begin{abstract}
A B S T R A C T
Background: Mediolateral balance impairment is an important cause of falling in older adults. We aimed to investigate whether hip abductor muscular strength and/or position sense are limiting factors in mediolateral balance control.

Methods: Sixteen community-dwelling older adults performed three different mediolateral weight-shifting tasks, by tracking (1) a sinusoidally moving visual target, "visual-MELBA"; (2) a sinusoidally translating platform without explicit visual feedback, "mechanical-MELBA"; and (3) an unpredictable platform translation, "sudden-platform-translation." Balance performance was quantified for each task and correlated with hip abductor position sense, isometric strength, and peak hip abduction/adduction moments and moment rates.

Findings: Participants with better balance performance showed higher and faster hip abduction/adduction moment production during the tasks. Isometric hip abductor strength was significantly correlated with accuracy of tracking the visual target, while hip abductor position sense was associated with the bandwidth over which the mechanical target could be tracked and with a smaller delay between CoM movement and the sudden-platform movement.

Interpretation: Hip abductor muscles play an important role in mediolateral balance control. Accurate balance performance appears limited by lower hip abductor strength when explicit visual information on balance reduces the need for hip abductor proprioception, while proprioceptive acuity may limit balance performance when no explicit enhanced feedback is presented and required weight shifts have to be inferred from "normal" sensory information.
\end{abstract}

(C) 2016 Elsevier Ltd. All rights reserved.

\section{Introduction}

On estimate, $30 \%$ of community-dwelling older adults fall at least once per year (Milat et al., 2011). Incorrect weight shifting is the most common cause of falling, and standing is one of the three classes of activities during which most falls in older adults occur (Robinovitch et al., 2013). Balance impairment, particularly in mediolateral (ML) direction, is suggested to be an important risk factor for such falls (Hilliard et al., 2008; Lord et al., 1999; Tinetti et al., 1988), and community-dwelling older adults are less able to actively control ML displacement of the center of mass (CoM) than young adults (Cofre Lizama et al., 2014).

ML-balance in bipedal stance is mainly controlled by a hip loadingunloading strategy through modulation of hip abductor muscle activity

\footnotetext{
* Corresponding author at: VU University Amsterdam, Van der Boechorststraat 9, 108 BT Amsterdam, the Netherlands.

E-mail address: j.van.dieen@vu.nl (J.H. van Dieën).
}

(Winter et al., 1993). An age-related reduction in hip abduction-adduction strength and torque production rate (Johnson et al., 2004; Kim et al., 2011) may negatively affect ML-balance control (Chang et al., 2005). Besides adequate muscle strength, balance control requires adequate integration of visual, vestibular, and somatosensory information (McCloskey, 1978). Hip abductor afference appears to play a role in this, since hip abductor vibration causes ML sway (Cofre Lizama et al., 2016; Roden-Reynolds et al., 2015). Although both the motor and the sensory capacity of the hip abductor musculature likely contribute to ML-balance control, it can be questioned whether these capacities limit balance performance in challenging situations.

We investigated whether hip abductor motor and sensory capacities limit balance control during predictable and unpredictable ML weight shifting in older adults. We correlated muscle strength and joint position sense with performance on weight-shifting tasks. As a visually guided task, we used a recently developed "MedioLateral Balance Assessment" tool (MELBA), in which participants track a visual target at increasing frequencies with ML movements of the CoM (Cofre 
Lizama et al., 2014). We additionally used a mechanical version of MELBA, without explicit visual feedback, in which target movements were imposed through platform translations that participants had to follow by shifting their CoM, again gradually increasing the frequency of target movements. Both of these tasks were continuous and predictable as target movements were sinusoidal. To compare, we also tested performance after transient unpredictable platform translations.

Because of the presence of explicit, enhanced visual feedback in visual-MELBA, proprioceptive information would be less important for balance (Cofre Lizama et al., 2016) and performance might consequently be limited by hip abductor strength. Hence, we hypothesized that people with higher isometric abductor strength will perform better on the visual-MELBA. Omitting explicit visual feedback, as in mechanical-MELBA and the unpredictable-platform-translation, makes proprioceptive information more important (Cofre Lizama et al., 2016), hence in these tasks, hip abductor proprioception might limit performance before hip strength does so. Therefore, we hypothesized hip proprioceptive acuity to be correlated to performance in mechanical-MELBA and after sudden-platform-translation.

\section{Methods}

\subsection{Participants}

Sixteen community-dwelling older adults (12 females and 4 males with a mean age of 68.3 (SD 4.8) years, a mean body height of 165.6 (SD 7.0$) \mathrm{cm}$, and a mean body mass of 68.1 (SD 12.6) $\mathrm{kg}$ ) participated in this study. Potential participants were excluded if they had a history of musculoskeletal disorders within the last 6 months, any pathology or previous surgery involving lower extremity joints or low back, or any neurological disorders including vestibular or visual problems and postural instability. The local ethics committee of KU Leuven approved the procedure. Participants signed informed consent before participation.

\subsection{Experimental design}

A novel mediolateral balance assessment task (MELBA) (Cofre Lizama et al., 2014), which consists of tracking a visual target with the body CoM, was used. To challenge various sensory and motor capacities, we selected (1) predictable weight shifting, tracking a sinusoidally moving visual target with explicit visual feedback, henceforth called "visual-MELBA"; (2) predictable weight shifting, by standing as still as possible on a sinusoidally translating platform without explicit visual feedback, henceforth called "mechanical-MELBA"; and (3) reactive unpredictable weight shifting to regain upright stance after a suddentransient platform translation to right or left, henceforth called "sudden-platform-translation". Body height was measured to standardize stance width during all tasks. Maximum hip abductor muscle strength and hip joint position sense (JPS) were assessed as measures of neuromuscular capacity.

The experiment always started with the visual- and the mechanicalMELBA (the order of the visual- and mechanical-MELBA was randomized between participants), followed by sudden-platform-translation. Four trials of visual-MELBA and mechanical-MELBA tasks were performed by each participant, of which the first one was used to get familiar with the task. For sudden-platform-translation, participants were given one practice trial before the measurements. Subsequently, eight trials were performed randomly ordered, yielding a total of four left and right translations.

\subsubsection{Visual-MELBA}

The visual-MELBA task has been described previously (Cofre Lizama et al., 2014). In short, participants were asked to stand with arms crossed, with a stance width of $11 \%$ of body height and a $14^{\circ}$ angle between the longitudinal axes of the feet, while their CoM position (red sphere of $9 \mathrm{~cm}$ diameter) was projected on a screen $(2 \times 1.5 \mathrm{~m}$ size) (Fig. 1). The projected target (white sphere of $11 \mathrm{~cm}$ diameter) moved sinusoidally in ML-direction, with an amplitude of $50 \%$ of stance width, while its movement frequency increased over time (Cofre Lizama et al., 2014). The target signal comprised 2 blocks of $20 \mathrm{~s}$ at 0.1 and $0.2 \mathrm{~Hz}, 1$ block of $10 \mathrm{~s}$ at $0.3 \mathrm{~Hz}$, and 13 blocks of $5 \mathrm{~s}$ at $0.4-1.6 \mathrm{~Hz}$ (with $0.1 \mathrm{~Hz}$ increments per block). The total duration of the task was $115 \mathrm{~s}$. The participants were instructed to track the target by ML-CoM movement as accurately as possible, by moving their entire body.

\subsubsection{Mechanical-MELBA}

The participants were asked to stand on a CAREN platform (Motekforce Link, Amsterdam, The Netherlands). The setup was the same as in Fig. 1, except that the representation of the CoM and the visual target were not projected. The target now was the mediolaterally moving platform; the subject was instructed to stand as still as possible to follow the platform translation. The target signal was constructed with the same frequency changes as the visual-MELBA, but the maximum amplitude of the platform movement, which was $50 \%$ of stance width, was decreased by $2 \%$ to $64 \%$ over the range from 0.4 to $1.6 \mathrm{~Hz}$, in view of the maximum acceleration of the CAREN platform.

\subsubsection{Sudden-platform-translation}

Participants were instructed to stand still in the same setup as used for MELBA and to regain upright stance as quickly as possible after a perturbation, without stepping. After a random interval of minimally $5 \mathrm{~s}$, the platform suddenly translated toward the left or right (Fig. 1). The

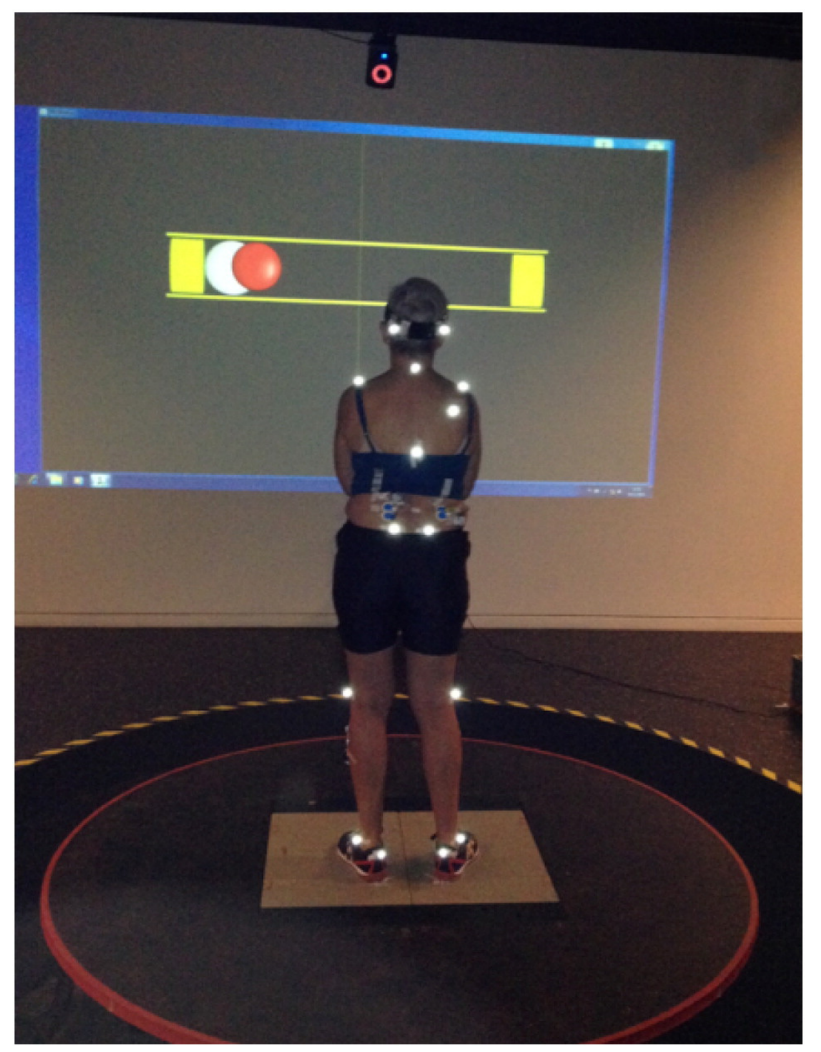

Fig. 1. The measurement setup for the weight-shifting tasks. The visual-MELBA is presented here on the screen (white sphere indicating the mediolaterally moving target to be tracked by the red sphere representing the body CoM). The mechanical-MELBA and sudden-translation tasks were assessed without any screen projection, but with the platform moving mediolaterally. 
mean velocity of the platform translation was $12 \mathrm{~cm} / \mathrm{s}$ and the amplitude was $10 \mathrm{~cm}$.

\subsubsection{Hip abduction strength}

Isometric hip abduction strength was assessed while standing on one leg and holding a horizontal bar placed in front of the body for external support. A force transducer (HBM, U2A, Darmstadt, Germany) was in series with a strap, attached by a velcro fixation around one of the ankles on one end and to a wall on the other end. Participants pulled the strap by abducting their extended leg with maximal force for $2 \mathrm{~s}$ and were not allowed to laterally bend their trunk during the test. Participants performed 3 trials with each leg, with sufficient time between trials. The force was sampled at 900 samples/s.

\subsubsection{Joint position sense (JPS)}

Participants stood blindfolded upright on both feet. For balance support, they were allowed to touch a horizontal bar located in front of them at hip height. The JPS for hip abduction was assessed with an "active-active" paradigm, i.e., participants (1) actively abducted the extended leg to a target orientation, identified by the examiner by the command "STOP," and responded "YES" when they had completely stopped upon which the examiner generated a trigger signal; (2) memorized the target orientation while holding their leg in that orientation for approximately $4 \mathrm{~s}$; (3) returned their leg to the starting orientation on the command "RETURN," and maintained that for 3-4 s; (4) actively reproduced the target position within $5 \mathrm{~s}$, again indicating the end of movement by saying "YES," to initiate a trigger set by the examiner. The target positions of the four trials for each leg were randomly varied within the range of $15-30^{\circ}$. The hip JPS test was also repeated for three trials for each leg. Subjects were allowed to rest between trials if desired. The intra-session reliability of this test was shown to be good to excellent (ICC 0.71-0.93) (Arvin et al., 2014).

\subsection{Data collection and preprocessing}

Twenty-three retro-reflective markers were attached bilaterally on the tip of the second toe, lateral/medial malleolus, heel, anterior tibia, lateral/medial femoral epicondyle, anterior thigh, anterior-/posteriorsuperior iliac crest, and acromion, and the forehead. Marker motion was captured by a 6-camera VICON system (6 MX camera system, VICON, Oxford Metrics, Oxford, UK) at a sample rate of 100 samples/s. Mechanical-MELBA was performed on the CAREN platform (Motekforce Link, Amsterdam, The Netherlands) with two embedded $0.8 \times 0.3 \mathrm{~m}$ force platforms (AMTI, Watertown, USA), sampling at 1000 samples/s.

Anatomical coordinate systems (ACS) were constructed for each segment, by making a short recording of the markers in static posture trials. The foot ACS was constructed based on the tip of the second toe, heel, lateral/medial malleolus. The shank ACS was defined by lateral and medial malleoli, and the anterior tibia condyle. The thigh ACS was based on the lateral and medial femoral epicondyles and the anterior aspect of the thigh. For the pelvis, the left and right anterior-superior iliac spines and posterior-superior iliac spines were used.

The body CoM was calculated with a 9-marker frontal plane model (forehead, acromions, anterior-superior iliac spines, lateral femoral epicondyles, and lateral malleoli). Sex-specific CoM calculations were performed according to De Leva (1996). D-flow 3.18.0 software (Motekforce Link, Amsterdam, The Netherlands) was used to produce target signals as well as to record and display target and CoM data at 100 sample/s.

\subsection{Data analysis}

\subsubsection{Visual and mechanical-MELBA}

Balance performance over the frequency range in the target signal was defined based on the linear constant coefficient transfer function between target signal and body CoM position, estimated using the
Welch algorithm over windows of 0.25 times the length of the target (per block) with $90 \%$ overlap between windows. For both the visualand mechanical-MELBA, the phase shift (PS; in degrees) and gain $(G)$ between target and CoM position were estimated. Perfect performance implies zero PS and G equal to one over all frequencies. The frequencies at which the PS dropped below $90^{\circ}$ and the G dropped below 0.5 were determined as the cutoff frequencies for phase shift $\left(f_{p s}\right)$ and gain $\left(\mathrm{f}_{\mathrm{G}}\right)$. The mean PS ( $\left.P S_{\text {mean }}\right)$ and mean $\mathrm{G}\left(\mathrm{G}_{\text {mean }}\right)$ were calculated over the bandwidth of $0.1 \mathrm{~Hz}$ to $\mathrm{f}_{\mathrm{ps}}$ and $\mathrm{f}_{\mathrm{G}}$, respectively (Cofre Lizama et al., 2014).

Besides these performance measures, the total net moment around the right and left hip joint in the frontal plane was calculated, applying a bottom-up inverse dynamics model (Kingma et al., 1996). This model calculates moments about a specified joint, based on external forces and segment kinematics and anthropometry. The model inputs in the present study were ground reaction forces, kinematics, and anthropometry of body segments below the hip joint (Hof, 1992). Center-of-pressure (CoP) measured by the force platform was corrected for platform motion in the mechanical-MELBA by adding the displacement of the ankle joint (stationary with respect to the force platform) to the CoP. The rate of the hip abduction/adduction moment was calculated as the first derivative of the hip abduction/adduction moment time series.

The peak hip abduction/adduction moments and moment rates were calculated over from the start of each trial until $\mathrm{f}_{\mathrm{ps}}$, and results for the left and right hips were averaged for statistical analyses. One subject was excluded for joint moment calculation as the force data were not appropriately saved during the measurement. All results were averaged over three trials. All data analyses were done using MATLAB R2012.

\subsubsection{Sudden-platform-translation}

After sudden-platform-translation, CoM movement lags behind platform movement (Fig. 2). Balance performance in this task was defined as time delay $\left(D_{\text {sud }}\right)$ between the CoM position and the platform position at $50 \%$ of the movement, with a smaller value indicating shorter delay and better balance performance.

The net abduction/adduction moments and the rates of moment changes around the left and right hip joint were calculated with correction for platform motion as described above. The peak abduction and adduction moments and the moment rates between translation onset and $2 \mathrm{~s}$ later were determined and averaged over trials and left and right hips.

\subsubsection{Hip abduction strength}

The force signal was low-pass filtered (cutoff frequency at $5 \mathrm{~Hz}$ ). Then, the maximum force over three trials was determined and multiplied by the length from anterior-superior iliac crest to medial malleolus, to calculate the isometric hip abduction moment. Left and right maximum hip abduction moments were averaged for further statistical analysis.

\subsubsection{JPS}

The hip abduction angle with respect to the pelvis segment was calculated according to (Wu et al., 2002). The target and reproduced hip abduction angles were averaged over a $400 \mathrm{~ms}$ interval around the trigger. The absolute difference between target angle and reproduced angle was defined as the absolute angular error $(\mathrm{AE})$. The $\mathrm{AE}$ represents the overall accuracy in performance in the JPS test. The AE was averaged over 4 trials and over left and right legs for further analysis (Arvin et al., 2014).

\subsubsection{Statistics}

Statistical analyses were performed in IBM SPSS Statistics 21.0 (IBM Corporation, Armonk, NY, USA). Descriptive statistics of balance performance are given as means and standard deviations of $\mathrm{f}_{\mathrm{ps}}, \mathrm{PS}_{\text {mean }}, \mathrm{f}_{\mathrm{G}}$, 

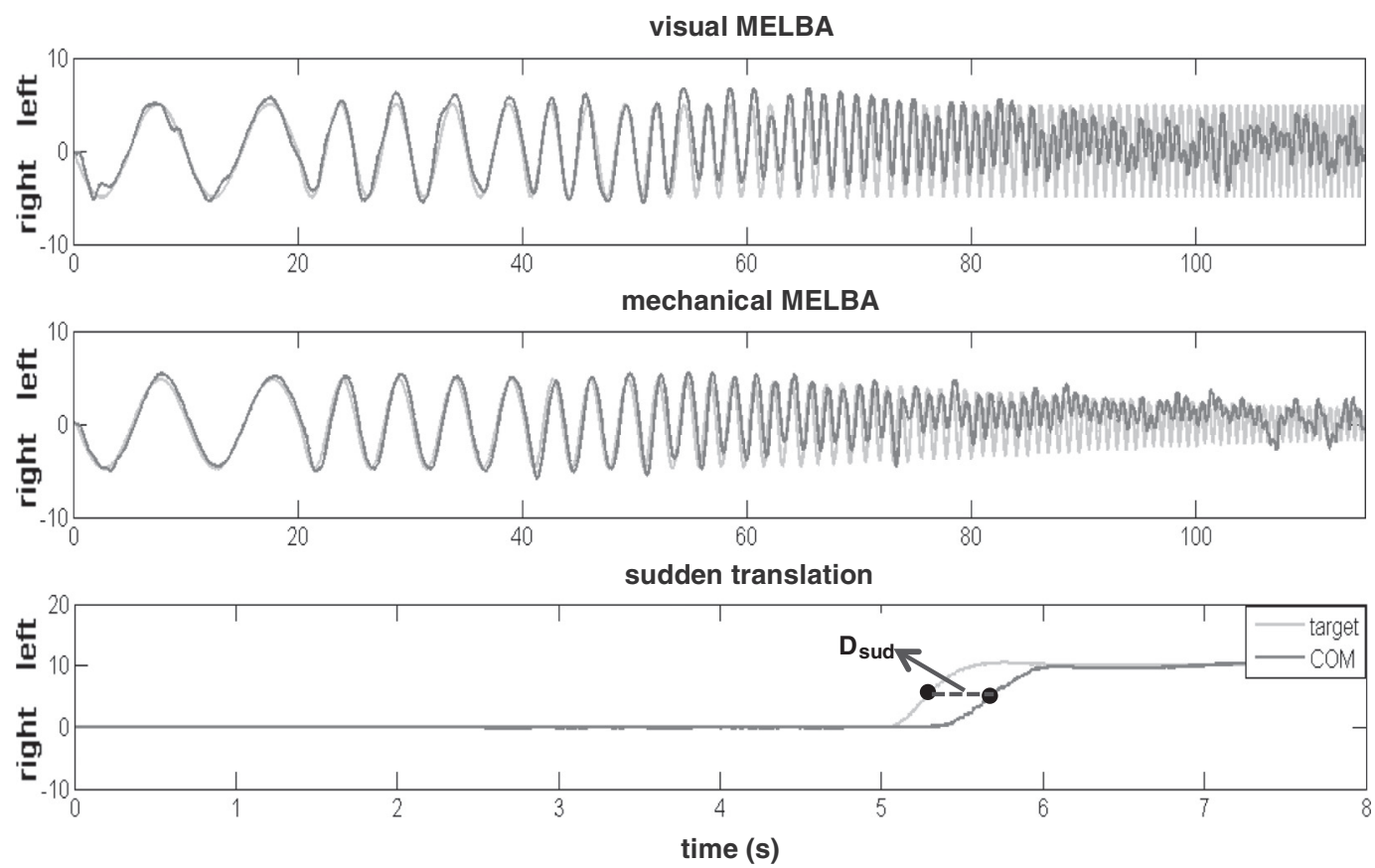

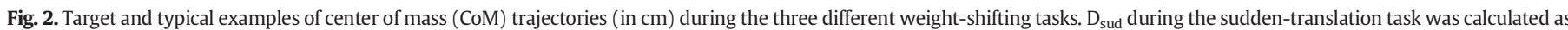
the time delay between the CoM position and the platform position at $50 \%$ of their movement (black circles)

$G_{\text {mean }}$, for visual and mechanical-MELBA and $D_{\text {sud }}$ for unpredictable platform translation.

Parametric assumptions were not violated. We compared balance performance measures ( $f_{P S}, P S_{\text {mean }}, f_{G}$ and $G_{\text {mean }}$ ) between visual and mechanical-MELBA by paired sample t-tests and peak hip abduction/ adduction moments and moment rates between all three tasks by one-way repeated-measures ANOVAs.

Pearson product-moment correlations were calculated to determine whether balance performance measures for both MELBA tasks and $\mathrm{D}_{\text {sud }}$ for the sudden-translation task were associated with (1) hip abduction/ adduction moments and moment rates during the tasks, (2) maximum isometric hip abduction strength, and (3) absolute errors in hip abduction repositioning. For all analyses, the significance level was set at 0.05 .

\section{Results}

\subsection{Neuromuscular capacity}

The mean absolute repositioning error and the mean isometric hip abduction strength over all participants were 1.72 (SD 0.97) ${ }^{\circ}$ and 83.40 (SD 35.05) Nm, respectively.

\subsection{Balance performance}

Balance performance measures of all weight-shifting tasks are presented in Table 1 . The mean phase difference between the target and the CoM was significantly lower during the visual-MELBA task than during the mechanical-MELBA task, indicating better tracking of the target during the visual-MELBA task in spite of the larger target signal amplitude. The other balance performance measures were comparable for the visual- and mechanical-MELBA tasks.

Typical examples of ML-hip moments during the three tasks illustrate that peak hip moments produced during the tasks were out of phase between left and right hips and increased to substantial levels only after $60 \mathrm{~s}$ in the MELBA tasks, i.e. at frequencies above $0.5 \mathrm{~Hz}$ (Fig. 3). The magnitudes of these peak moments and moment rates within the bandwidth were not significantly different between tasks at group level (Table 1 ).

\subsection{Correlations between performance and moments produced during the tasks}

For the visual-MELBA task, the performance measure $\mathrm{f}_{\mathrm{ps}}$ was significantly correlated with peak hip adduction and abduction moments (Table 2), indicating that higher hip moments are produced when the target is tracked to higher frequencies with limited phase lag. Similar results were obtained for hip moment rates (Table 2). For the other performance indicators, no significant correlations with hip moment and moment rates were found.

For the mechanical-MELBA task, the performance measures $\mathrm{f}_{\mathrm{ps}}$ and $\mathrm{PS}_{\text {mean }}$ were significantly correlated with peak hip adduction and abduction moments and moment rates (Table 2), indicating that greater and faster hip moments are produced when the target is tracked with a limited phase lag to higher frequencies. Also the correlations between $\mathrm{G}_{\text {mean }}$ and peak hip abduction and adduction moments were significant (Table 2), indicating that greater hip moments are produced when higher levels of $\mathrm{G}_{\text {mean }}$ are reached.

Table 1

Balance performance measures ( $\mathrm{f}_{\mathrm{ps}}, \mathrm{PS}_{\text {mean }}, \mathrm{f}_{\mathrm{G}}$, and $\mathrm{G}_{\text {mean, }}$ see text) and peak hip abduction/ adduction moments and moment rates (mean (SD)) during visual- and mechanical-MELBA tasks and the sudden-translation task. P-values indicate condition effects.

\begin{tabular}{llllr}
\hline & Visual-MELBA & Mechanical-MELBA & Sudden-translation & $p$-value \\
\hline $\mathrm{f}_{\mathrm{ps}}(\mathrm{Hz})$ & $0.82(0.20)$ & $0.87(0.19)$ & $\mathrm{N} / \mathrm{A}$ & 0.582 \\
$\mathrm{PS}_{\text {mean }}\left({ }^{\circ}\right)$ & $-34.36(5.35)$ & $-48.60(6.89)$ & $\mathrm{N} / \mathrm{A}$ & $<0.001$ \\
$\mathrm{f}_{\mathrm{G}}$ & $0.82(0.17)$ & $0.74(0.12)$ & $\mathrm{N} / \mathrm{A}$ & 0.226 \\
$\mathrm{G}_{\text {mean }}$ & $0.79(0.05)$ & $0.75(0.14)$ & $\mathrm{N} / \mathrm{A}$ & 0.268 \\
$\mathrm{D}_{\text {sud }}(\mathrm{s})$ & $\mathrm{N} / \mathrm{A}$ & $\mathrm{N} / \mathrm{A}$ & $0.50(\mathrm{SD} 0.12)$ & $\mathrm{N} / \mathrm{A}$ \\
$\begin{array}{c}\text { Add moment } \\
\quad(\mathrm{Nm})\end{array}$ & $22.25(7.18)$ & $21.59(7.44)$ & $21.01(1.71)$ & 0.633 \\
$\begin{array}{c}\text { Abd moment } \\
\quad(\mathrm{Nm})\end{array}$ & $30.17(10.87)$ & $29.15(10.17)$ & $35.23(10.26)$ & 0.099 \\
$\begin{array}{c}\text { Add moment } \\
\text { rate }(\mathrm{Nm} / \mathrm{s})\end{array}$ & $157.53(79.46)$ & $130.97(82.13)$ & $137.74(28.16)$ & 0.439 \\
$\begin{array}{c}\text { Abd moment } \\
\text { rate }(\mathrm{Nm} / \mathrm{s})\end{array}$ & $159.47(74.53)$ & $129.89(84.98)$ & $135.35(19.86)$ & 0.373 \\
\hline
\end{tabular}



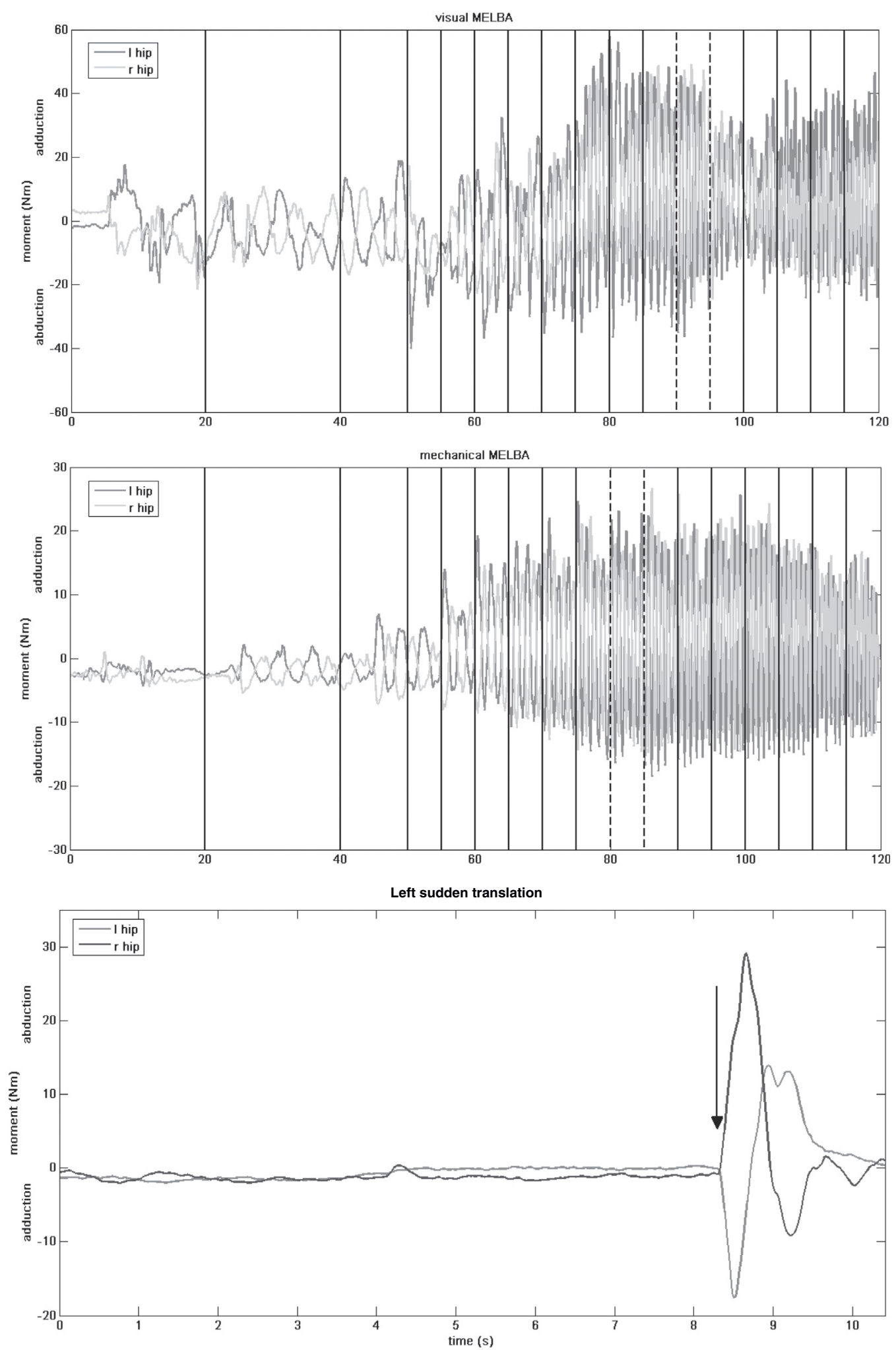

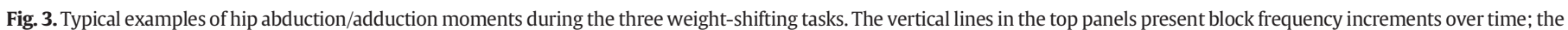
dashed vertical lines indicate the block where the performance dropped. The arrow in the bottom panel represents the platform translation onset.

For the sudden-translation task, $\mathrm{D}_{\text {sud }}$ was significantly and negatively correlated with the peak hip abduction and adduction moments (Table 2), indicating that participants producing greater hip moments showed a smaller delay of CoM to platform movement.

\subsection{Correlations between performance and neuromuscular capacity}

For the visual-MELBA task, only isometric hip abduction strength correlated with performance, in particular, $\mathrm{PS}_{\text {mean }}$ and $\mathrm{G}_{\text {mean }}$, were significantly and positively correlated with maximum hip abduction 
Table 2

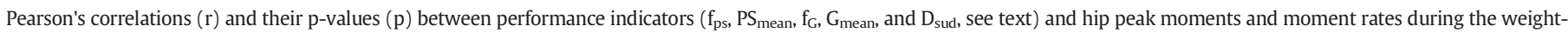
shifting tasks. Significant correlations are printed in bold.

\begin{tabular}{|c|c|c|c|c|c|c|c|c|c|}
\hline & & \multicolumn{2}{|c|}{$\begin{array}{l}\text { Hip adduction } \\
\text { moment }\end{array}$} & \multicolumn{2}{|c|}{ Hip abduction moment } & \multicolumn{2}{|c|}{$\begin{array}{l}\text { Hip adduction moment } \\
\text { rate }\end{array}$} & \multicolumn{2}{|c|}{$\begin{array}{l}\text { Hip abduction moment } \\
\text { rate }\end{array}$} \\
\hline & & $r$ & $p$ & $r$ & $p$ & $r$ & $p$ & $r$ & $p$ \\
\hline Visual- & $\mathrm{f}_{\mathrm{ps}}$ & 0.73 & 0.002 & 0.73 & 0.002 & 0.90 & $<0.001$ & 0.87 & $<0.001$ \\
\hline \multirow[t]{3}{*}{ MELBA } & $\mathrm{PS}_{\text {mean }}$ & 0.36 & 0.178 & 0.30 & 0.279 & 0.40 & 0.136 & 0.37 & 0.180 \\
\hline & $\mathrm{f}_{\mathrm{G}}$ & 0.35 & 0.199 & 0.168 & 0.551 & 0.30 & 0.271 & 0.32 & 0.249 \\
\hline & $\mathrm{G}_{\text {mean }}$ & 0.03 & 0.907 & 0.02 & 0.942 & 0.08 & 0.766 & 0.05 & 0.839 \\
\hline \multirow[t]{4}{*}{ Mechanical-MELBA } & $\mathrm{f}_{\mathrm{ps}}$ & 0.63 & 0.012 & 0.54 & 0.038 & 0.62 & 0.013 & 0.65 & 0.008 \\
\hline & $\mathrm{PS}_{\text {mean }}$ & 0.66 & 0.008 & 0.59 & 0.021 & 0.64 & 0.010 & 0.63 & 0.011 \\
\hline & $\mathrm{f}_{\mathrm{G}}$ & 0.15 & 0.583 & 0.40 & 0.139 & 0.08 & 0.770 & 0.03 & 0.901 \\
\hline & $\mathrm{G}_{\text {mean }}$ & 0.68 & 0.005 & 0.68 & 0.005 & 0.49 & 0.063 & 0.50 & 0.059 \\
\hline Sudden-translation & $\mathrm{D}_{\text {sud }}$ & -0.73 & 0.003 & -0.56 & 0.037 & -0.22 & 0.451 & -0.16 & 0.599 \\
\hline
\end{tabular}

strength (Table 3). For the mechanical-MELBA task, $\mathrm{f}_{\mathrm{ps}}$ correlated with $\mathrm{AE}$ in the JPS test (Table 3), indicating that participants with lower errors in position sense are those who tracked the target up to a higher frequency of the target movement. AE was also correlated with time delay $\left(D_{\text {sud }}\right)$ in the sudden-translation task, implying that participants with more accurate hip position sense are those who had shorter delay in tracking the platform translation.

\section{Discussion}

The present study was aimed to determine whether the neuromuscular capacity of the hip abductor muscles is associated with ML-weight balance control in older adults. To do so, our participants performed three different ML-weight-shifting tasks. Higher hip moments and moment rates produced during all tasks were correlated with better performance, indicating that hip moment production was required in these tasks. We correlated performance on the weight-shifting tasks to hip abductor neuromuscular capacity, i.e. their maximum muscle strength and acuity of hip joint position sense. In line with our hypotheses, participants with higher maximum isometric hip abductor strength tracked the visual target more accurately, while participants who made smaller repositioning errors could track the mechanical target up to higher frequencies and could track the unpredictable platform movement with a shorter time delay.

Impaired ML-balance control in standing has been identified as a common cause of falls among older adults (Robinovitch et al., 2013) and hip abductor strength is predictive of future falls (Hilliard et al., 2008). We therefore suggested that hip abductor strength might limit ML-weight-shifting performance. The results of the present study offer partial support for this notion; we showed that higher and faster hip abductor/adductor moment production was associated with better performance in terms of a larger bandwidth and more accurate tracking of predictable target movements with the CoM and in terms of faster reactive control after sudden unexpected perturbations. Although this

Table 3

Pearson's correlation ( $r$ ) and their p-values (p) between performance indicators $\left(f_{p s}\right.$, $\mathrm{PS}_{\text {mean }}, \mathrm{f}_{\mathrm{G}}, \mathrm{G}_{\text {mean }}$, and $\mathrm{D}_{\text {sud, }}$, see text) in the weight-shifting tasks, absolute error of joint position sense test and maximum isometric hip moment. Significant correlations are highlighted in bold.

\begin{tabular}{|c|c|c|c|c|c|}
\hline & & \multicolumn{2}{|c|}{$\begin{array}{l}\text { Isometric hip } \\
\text { abduction strength }\end{array}$} & \multicolumn{2}{|c|}{ Absolute error } \\
\hline & & $r$ & $p$ & $r$ & $p$ \\
\hline \multirow[t]{4}{*}{ Visual-MELBA } & $\mathrm{f}_{\mathrm{ps}}$ & 0.20 & 0.486 & 0.30 & 0.285 \\
\hline & $\mathrm{PS}_{\text {mean }}$ & 0.60 & 0.023 & 0.26 & 0.355 \\
\hline & $\mathrm{f}_{\mathrm{G}}$ & 0.244 & 0.400 & 0.10 & 0.698 \\
\hline & $\mathrm{G}_{\text {mean }}$ & 0.55 & 0.041 & 0.10 & 0.716 \\
\hline \multirow[t]{4}{*}{ Mechanical-MELBA } & $\mathrm{f}_{\mathrm{ps}}$ & -0.08 & 0.775 & -0.50 & 0.048 \\
\hline & $\mathrm{PS}_{\text {mean }}$ & -0.13 & 0.657 & -0.29 & 0.292 \\
\hline & $\mathrm{f}_{\mathrm{G}}$ & 0.147 & 0.615 & -0.09 & 0.733 \\
\hline & $\mathrm{G}_{\text {mean }}$ & -0.11 & 0.691 & -0.35 & 0.194 \\
\hline Sudden-translation & $\mathrm{D}_{\text {sud }}$ & -0.12 & 0.701 & 0.63 & 0.016 \\
\hline
\end{tabular}

implies that ML weight shifting does require hip moment production, it does not necessarily imply that hip abductor strength is the limiting factor for ML-balance control. In fact, the findings revealed that maximum isometric hip abductor strength was correlated with $\mathrm{PS}_{\text {mean }}$ and $\mathrm{G}_{\text {mean }}$ in the visual-MELBA task, indicating that it may limit accuracy of performance, but not bandwidth (reflected in $\mathrm{f}_{\mathrm{PS}}$ and $\mathrm{f}_{\mathrm{G}}$ ). It should be noted that the peak moments produced during all tasks were well below the maximum isometric strength $(<50 \%)$, but this could be a consequence of the dynamic nature of the task. Moreover, no correlations were found between maximum isometric hip abduction strength and peak moments or rates of moment development during the MELBA tasks. Strength was tested isometrically after a steady increase in muscle force. In contrast, during the weight-shifting tasks, participants produced quickly alternating hip moments. This may also explain why no correlations were found between maximum isometric hip abduction strength and peak moments or rates of moment development during the MELBA tasks. Future studies could investigate whether the maximum rate of (abduction) force development is more predictive for dynamic balance performance.

Besides sufficient muscle strength, adequate scaling of CoM movements in weight shifting likely requires accurate online sensory information regarding CoM position and velocity. It has been proposed that visual and proprioceptive inputs are the main sensory feedback modalities to maintain balance in standing (Fitzpatrick et al., 1994) and that older adults rely more on visual feedback than young adults (Hay et al., 1996). However, unilateral hip abductor muscle vibration, which affects proprioceptive afference from the muscle spindles, resulted in contralateral postural sway indicating that hip abductor proprioception is important for control of standing posture (Cofre Lizama et al., 2016; Roden-Reynolds et al., 2015). The mechanical-MELBA and suddentranslation tasks were hypothesized to be more dependent on proprioceptive information than the visual task, as required weight shifts had to be inferred from sensory information and no explicit visual instruction and feedback might attenuate the effect of proprioceptive input in the control of upright stance (Cofre Lizama et al., 2016). As discussed above, in the visual-MELBA task, where explicit visual feedback on CoM movement was presented, isometric hip abduction strength and not JPS correlated with performance. In contrast, lower proprioceptive acuity coincided with an increase in phase lag between CoM and platform movement at lower frequencies in the mechanical-MELBA, and with an increase in time delay between platform and CoM movement in the sudden-translation task. The results suggest that visual-MELBA is easier than mechanical-MELBA, as indicated by the lower phase shift. Probably performance is limited earlier in mechanical-MELBA due to the fact that proprioceptive information is more needed to control the CoM movements in relation to the target. In visual-MELBA, this limiting factor would be by-passed by the availability of explicit, enhanced feedback, hence performance is better until it is limited by muscle strength. In line with this, consistently higher, albeit nonsignificantly, average moments and moment rates were reached in visual- than in mechanical-MELBA. The MELBA tasks most likely 
rely on both feedforward and feedback control mechanisms, given the predictable nature of the target movements. In comparison, the sudden-translation task heavily relies on feedback control, which may explain the stronger correlation with JPS (Table 3). Therefore, performance in these tasks appears to be determined by several factors, with the main limiting factor being different between tasks.

The findings of this study indicate that improving hip abductor neuromuscular capacity through rehabilitation programs might improve balance control and subsequently decrease fall risk, especially in older adults with impaired hip abductor muscles (e.g., older adults after stroke or hip surgery). However, it should be noted that neuromuscular capacity of the hip abductor muscles, i.e. muscle strength and JPS, was only moderately and not consistently correlated with balance performance measures in the weight-shifting tasks, indicating that these are not the sole limiting factors in ML-balance control. For example, the capacity of other muscle groups such as ankle invertors/evertors and of the vestibular system might be additional determinants of control during weight shifting in the ML-direction. Therefore, further study is required to provide the clinic with evidence-based advice regarding the optimal rehabilitation program to improve ML-balance control. According to the findings of this study, hip adductor moment/moment rates were also correlated to ML-balance control in ML-weight-shifting tasks. As we did not measure isometric hip adductor strength, further study on hip adductor neuromuscular capacity as a possible limiting factor in ML-balance control may complement the present findings.

\section{Conclusion}

The results of the present study support the notion that hip abductor muscles play an important role in mediolateral balance control. Maximum hip abductor strength was associated with accurate balance performance when explicit visual feedback on balance was provided, while hip abductor proprioception acuity was associated with balance performance in expected and unexpected platform translations, when required weight shifts had to be inferred from sensory information and no explicit visual instruction and feedback were presented.

\section{Acknowledgment}

This work was supported by the European Commission through MOVE-AGE, an Erasmus Mundus Joint Doctorate program (grant number 2011-0015). Mirjam Pijnappels was financially supported by a VIDI grant (grant number 91714344) from the Dutch Organization for Scientific Research (NWO).

\section{References}

Arvin, M., Hoozemans, M.J., Burger, B.J., Verschueren, S.M., van Dieën, J.H., Pijnappels, M., 2014. Reproducibility of a knee and hip proprioception test in healthy older adults. Aging Clin. Exp. Res. 27, 171-177.

Chang, S.-H.J., Mercer, V.S., Giuliani, C.A., Sloane, P.D., 2005. Relationship between hip abductor rate of force development and mediolateral stability in older adults. Arch. Phys. Med. Rehabil. 86, 1843-1850.

Cofre Lizama, L.E., Pijnappels, M., Faber, G.H., Reeves, P.N., Verschueren, S.M., van Dieen, J.H., 2014. Age effects on mediolateral balance control. PLoS One 9, e110757.

Cofre Lizama, L.E., Pijnappels, M., Reeves, N.P., Verschueren, S.M., van Dieën, J.H., 2016. Can explicit visual feedback of postural sway efface the effects of sensory manipulations on mediolateral balance performance? J. Neurophysiol. 115, 907-914.

De Leva, P., 1996. Adjustments to zatsiorsky-seluyanov's segment inertia parameters. J. Biomech. 29, 1223-1230.

Fitzpatrick, R., Rogers, D.K., McCloskey, D., 1994. Stable human standing with lower-limb muscle afferents providing the only sensory input. J. Physiol. 480, 395-403.

Hay, L., Bard, C., Fleury, M., Teasdale, N., 1996. Availability of visual and proprioceptive afferent messages and postural control in elderly adults. Exp. Brain Res. 108, 129-139.

Hilliard, M.J., Martinez, K.M., Janssen, I., Edwards, B., Mille, M.-L., Zhang, Y., Rogers, M.W., 2008. Lateral balance factors predict future falls in community-living older adults. Arch. Phys. Med. Rehabil. 89, 1708-1713.

Hof, A.L., 1992. An explicit expression for the moment in multibody systems. J. Biomech. $25,1209-1211$.

Johnson, M.E., Mille, M.-L., Martinez, K.M., Crombie, G., Rogers, M.W., 2004. Age-related changes in hip abductor and adductor joint torques. Arch. Phys. Med. Rehabil. 85, 593-597.

Kim, J.W., Kwon, Y., Chung, H.Y., Eom, G.M., Jun, J.H., Chung, J.S., Park, B.K., 2011. Age-sex differences in the hip abductor muscle properties. Geriatr. Gerontol. Int. 11, 333-340.

Kingma, I., de Looze, M.P., Toussaint, H.M., Klijnsma, H.G., Bruijnen, T.B., 1996. Validation of a full body 3-d dynamic linked segment model. Hum. Mov. Sci. 15, 833-860.

Lord, S.R., Rogers, M.W., Howland, A., Fitzpatrick, R., 1999. Lateral stability, sensorimotor function and falls in older people. J. Am. Geriatr. Soc.

McCloskey, D., 1978. Kinesthetic sensibility. Physiol. Rev. 58, 763-820.

Milat, A.J., Watson, W.L., Monger, C., Barr, M., Giffin, M., Reid, M., 2011. Prevalence, circumstances and consequences of falls among community-dwelling older people: results of the 2009 nsw falls prevention baseline survey. N. S. W. Public Health Bull. 22, 43-48.

Robinovitch, S.N., Feldman, F., Yang, Y., Schonnop, R., Leung, P.M., Sarraf, T., Sims-Gould, J., Loughin, M., 2013. Video capture of the circumstances of falls in elderly people residing in long-term care: an observational study. Lancet 381, 47-54.

Roden-Reynolds, D.C., Walker, M.H., Wasserman, C.R., Dean, J.C., 2015. Hip proprioceptive feedback influences the control of mediolateral stability during human walking. J. Neurophysiol. 114, 2220-2229.

Tinetti, M.E., Speechley, M., Ginter, S.F., 1988. Risk factors for falls among elderly persons living in the community. N. Engl. J. Med. 319, 1701-1707.

Winter, D., Prince, F., Stergiou, P., Powell, C., 1993. Medial-lateral and anterior-posterior motor-responses associated with center of pressure changes in quiet standing. Neurosci. Res. Commun. 12, 141-148.

Wu, G., Siegler, S., Allard, P., Kirtley, C., Leardini, A., Rosenbaum, D., Whittle, M., D'Lima, D.D., Cristofolini, L., Witte, H., 2002. Isb recommendation on definitions of joint coordinate system of various joints for the reporting of human joint motion-part i: ankle, hip, and spine. J. Biomech. 35, 543-548. 